\title{
Influence of colour Doppler echocardiography on the ultrasonic assessment of congenital heart disease: a prospective study
}

Department of Cardiology, Royal Hospital for Sick Children, Glasgow J P Gnanapragasam A B Houston W B Doig R Fraser $S$ Lilley

E Murtagh G Olafsson

Correspondence to Dr J P Gnanapragasam, Department of Cardiology, Royal Hospital for Sick Children, Glasgow G3 8SJ. Accepted for publication 11 April 1991

\author{
J P Gnanapragasam, A B Houston, W B Doig, R Fraser, S Lilley, E Murtagh, G Olafsson
}

\begin{abstract}
Objective-To evaluate the additional information provided by colour Doppler in the ultrasonic assessment of congenital heart disease.
\end{abstract}

Patients and methods-A prospective study of 215 children (age range 1 day-16 years) presenting with clinical signs of congenital heart disease.

Results-Colour Doppler was essential for the diagnosis of an anomalous left coronary artery and altered the management of a patient initially diagnosed as having cardiomyopathy. Colour Doppler provided extra information, but without major impact on management, in the following: the diagnosis of ventricular septal defects associated with other defects, of multiple ventricular septal defects, of anomalous pulmonary venous drainage, and of mild mitral regurgitation; the demonstration of site of coarctation, of stenotic or hypoplastic pulmonary artery branches, of unobstructed flow through a right atrial membrane, and of left ventricle to right atrium regurgitation; the assessment of the width of the duct and of flow through the patent foramen ovale in transposition and tricuspid atresia; the differentiation of pulmonary atresia from critical pulmonary stenosis and the measurement of maximum velocity of tricuspid regurgitation.

Conclusions-Ideally all patients should undergo colour Doppler studies before cardiac surgery to ensure a more accurate diagnosis. However, since the additional information provided does not affect the management in most patients, machines without colour Doppler can provide a satisfactory service in paediatric cardiology centres in countries where resources are limited.

Cross sectional imaging of cardiac structures and spectral Doppler examination of cardiac flow pattern $\mathrm{s}^{2}$ can provide an accurate assessment of most congenital cardiac defects. While spectral pulsed wave Doppler displays the velocity of only a single sample volume, colour Doppler provides a graphic display of flow velocity profiles superimposed over an area of the cross sectional image. Because this can facilitate the recognition of abnormal flow and of correlation with the underlying structural abnormality the use of colour coded Doppler velocity mapping should increase the diagnostic potential of cardiac ultrasound. ${ }^{3}$ However, there have been no prospective studies to ascertain the influence of colour Doppler on the final non-invasive assessment of congenital heart disease. This information would be very useful in centres where some studies are still performed on machines without colour Doppler and is particularly relevant in the current period of worldwide expansion in paediatric cardiology for centres with limited financial resources. Knowledge of the capabilities and limitations of the techniques is important because most infants and children with congenital heart disease are now referred for surgery on the basis of noninvasive investigations. ${ }^{45} \mathrm{We}$ therefore carried out a prospective study to assess the additional information provided by colour Doppler in the non-invasive assessment of congenital heart disease.

\section{Patients and methods}

PATIENTS

We studied 215 patients (age range 1 day to 16 years) presenting with clinical signs of congenital heart disease. There were 84 neonates, 54 older infants, and 77 older children. The patients were studied at initial presentation and their diagnoses are listed in the table. In order to perform a detailed ultrasound examination on patients enrolled in the study without disrupting the work of the department some new patients could not be enrolled in the study during busy clinic sessions. Patients were excluded purely because machine time was not available and not on the basis of medical criteria.

\section{METHODS}

The studies were performed by a doctor or an experienced technician who was aware of the provisional diagnosis. A detailed cross sectional study was performed first. This was followed by Doppler interrogation with nonimage linked (stand alone) continuous wave Doppler and image linked (duplex) continuous and pulsed wave Doppler. When the operator was satisfied that all possible information had been obtained with each technique the findings were recorded in detail. Colour Doppler examination was then carried out and the additional information obtained by this 
List of diagnoses with the total number of defects in patients, number who were catheterised before surgery or during interventional procedures, and number treated by palliative or corrective surgery

\begin{tabular}{|c|c|c|c|}
\hline Diagnosis & Total & Catheterised & Operated \\
\hline \multicolumn{4}{|l|}{ Ventricular septal defect: } \\
\hline Single isolated & 51 & 1 & 2 \\
\hline Single with other defect & 49 & - & - \\
\hline Multiple & 6 & 0 & 0 \\
\hline \multicolumn{4}{|l|}{ Patent ductus arteriosus: } \\
\hline Isolated & 14 & $1^{\star}$ & 3 \\
\hline With other defect & 49 & - & - \\
\hline \multicolumn{4}{|l|}{ Atrial septal defect: } \\
\hline Secundum & 27 & 1 & 9 \\
\hline Sinus venosus & 1 & 0 & 1 \\
\hline Atrioventricular septal defect & 14 & 1 & 10 \\
\hline Pulmonary valve stenosis & 11 & $2^{\star}$ & 0 \\
\hline Subpulmonary stenosis & 6 & 2 & 2 \\
\hline Pulmonary artery stenosis & 4 & 2 & 2 \\
\hline Tetralogy of Fallot & 10 & 3 & $4(1+)$ \\
\hline Pulmonary atresia with VSD & 3 & 0 & $3+$ \\
\hline Pulmonary atresia with intact septum & 4 & 0 & $4 \dagger$ \\
\hline Double outlet right ventricle & 11 & 1 & $9 \dagger$ \\
\hline Aortic valve stenosis & 11 & 0 & 1 \\
\hline Subaortic stenosis & 4 & 0 & 3 \\
\hline Supra-aortic valve stenosis & 1 & 0 & 1 \\
\hline Coarctation & 17 & 1 & 17 \\
\hline Hypoplastic left heart & 6 & 0 & 6 \\
\hline Transposition & 11 & 0 & 11 \\
\hline Tricuspid atresia & 3 & 0 & $3 \dagger$ \\
\hline Double inlet ventricle & 2 & 0 & $2 \dagger$ \\
\hline Mitral regurgitation/stenosis & 3 & 0 & 1 \\
\hline Anomalous left coronary artery & 1 & 0 & 1 \\
\hline Dilated cardiomyopathy & 3 & 0 & 0 \\
\hline Common arterial trunk & 1 & 0 & 1 \\
\hline Common arterial trunk with IAA & 1 & 0 & 0 \\
\hline Aortic origin of pulmonary artery & 1 & 0 & 1 \\
\hline TAPVD & 1 & 0 & 1 \\
\hline Cor triatriatum & 1 & 0 & 1 \\
\hline Cor triatriatum dexter & 1 & 0 & 1 \\
\hline
\end{tabular}

ॠInterventional catheterisation. +Palliative surgery.

IAA, interrupted aortic arch; TAPVD, total anomalous pulmonary venous drainage; VSD, ventricular septal defect. ening previously available information. We studied patients only at the initial examination, and the role of colour Doppler in the postoperative evaluation of patients was not assessed.

\section{Results}

To simplify reporting of the assessment of the results we considered the findings under the diagnostic categories determined by the final non-invasive diagnosis. The total number of defects analysed exceeded the number of patients in the study because many patients had more than one defect.

VENTRICULAR SEPTAL DEFECT $(\mathrm{n}=106)$

Single isolated ventricular septal defects $(n=51)$

Defect localisation-The presence of flow through a ventricular septal defect, but not its site, was shown in all 51 cases by the nonimaging continuous wave transducer. Cross sectional echocardiography successfully showed the site of a defect in only 28 of the 51 patients with a single uncomplicated lesion, including two in whom the defect appeared to be closed by tricuspid valve tissue. However, image linked high pulse repetition frequency Doppler echocardiography identified the approximate site of the ventricular septal defect in 18 of the 23 patients in whom the defect could not be seen on imaging, and colour Doppler showed the exact site of defect in all 23 patients. The five defects whose site could be located only with the use of colour Doppler were very small and situated in the trabecular $(\mathbf{n}=2)$ and apical $(\mathbf{n}=3)$ muscular septum. In the two patients with apparent closure of a perimembranous ventricular septal defect by tricuspid valve tissue colour Doppler showed the site of flow through the aneurysm.

Estimation of right ventricular pressure-The pressure drop across the ventricular septal defect estimated by aligning the Doppler beam along the direction of the colour jet was more than $10 \mathrm{~mm} \mathrm{Hg}$ greater than that estimated without colour Doppler in only two patients (85-96 mm Hg, 40-52 mm Hg). The spectral signal of tricuspid regurgitation was sought in every patient and a satisfactory signal showing the peak velocity from which the right ventricular pressure could be estimated was obtained in 17 of the 51 patients. The use of colour Doppler to localise the flow of tricuspid regurgitation improved the spectral waveforms and enabled the maximum velocity to be measured in four additional patients.

Single ventricular septal defect with other defects $(n=49)$

This group had ventricular septal defects associated with other defects but not atrioventricular septal defects which are considered separately. The presence of a ventricular septal defect was detected without the use of colour Doppler in 44 patients. The remaining five had very small muscular defects the presence of which was unsuspected until colour Doppler certainty of a diagnosis. To make the study objective as possible and to eliminate any possible bias we confined our study to the finding of additional information and did not assess the role of colour Doppler in strength- 
was used (pulmonary stenosis 3 , transposition 1, coarctation 1 ).

Multiple ventricular septal defect $(n=6)$ Colour Doppler identified two areas of flow through the ventricular septum in six patients. Cross sectional imaging showed both defects in only two patients. Spectral Doppler detected a signal of ventricular septal defect flow in all patients, but in four patients the presence of more than one defect was not recognised until colour Doppler was used.

ATRIAL SEPTAL DEFECT $(\mathrm{n}=28)$

There were 27 patients with a secundum atrial septal defect, two of which were fenestrated defects, and one patient with a sinus venosus defect. Imaging studies showed the defect in the atrial septum in every patient and the anomalous site of entry of the right lower pulmonary vein in the patient with an inferior sinus venosus defect. However, in another child with a secundum defect, anomalous drainage of the right pulmonary veins into the inferior vena cava was unsuspected until colour Doppler echocardiography showed an unusual flow signal entering the inferior vena cava near its junction with the right atrium.

We obtained a complete spectral signal of tricuspid regurgitation that enabled its maximum velocity to be measured in 10 patients without the use of colour Doppler; in one additional patient the maximum velocity was measured by using colour.

\section{ATRIOVENTRICULAR SEPTAL DEFECT $(n=14)$}

Cross sectional imaging clearly showed the atrial component of the defect in all patients and a ventricular septal defect in 11 patients. Colour Doppler showed a very small ventricular septal defect at the crest of the septum in an additional patient. This ventricular septal defect could not be detected by spectral Doppler because the flow signal was obscured by atrioventricular valve regurgitation which arose from the central part of the valve. Cross sectional imaging showed a common valve in five patients and two separate valve components in nine. Spectral Doppler detected atrioventricular valve regurgitant flow on the left side in 13 patients and on the right side in 10, and left ventricle to right atrium regurgitant flow was suspected on duplex Doppler in one patient. This was confirmed by colour Doppler, which also showed similar left ventricle to right atrium flow in six other patients.

\section{PULMONARY STENOSIS AND TETRALOGY OF} FALLOT $(\mathrm{n}=31)$

Colour Doppler provided information on pulmonary artery branch anatomy in five patients in whom this was not obtained by imaging owing to obscuring by lungs. It defined the size of a very hypoplastic left pulmonary artery $(n=1)$ and showed normal sized distal pulmonary artery branches $(n=2)$ in patients with tetralogy of Fallot. In a patient with isolated distal left pulmonary artery stenosis colour Doppler showed the site of narrowing which could not be seen on imaging. In another patient with normal sized right and left central pulmonary arteries colour Doppler showed areas of turbulence more peripherally in the lungs, indicating the presence of peripheral pulmonary artery stenoses.

A neonate with double outlet right ventricle and narrowing of the right ventricular outflow tract had a very dysplastic pulmonary valve that seemed to have a small orifice, but flow through it was difficult to demonstrate by duplex Doppler owing to interference by flow from a large duct. Colour Doppler showed an area of flow through the tiny orifice of the valve that was distinct from the ductal jet into the pulmonary artery.

Colour Doppler did not provide any additional information in the other patients in terms of defining the anatomy or estimating the maximum velocity across the stenosis.

PULMONARY ATRESIA WITH AND WITHOUT VENTRICULAR SEPTAL DEFECT $(\mathbf{n}=7)$

Colour Doppler had no influence on the detection of confluence of pulmonary artery branches, presence of a main pulmonary artery, or on the measurement of proximal pulmonary artery branch size. In two patients the pulmonary artery branches were seen more distally with the use of colour Doppler. Pulmonary valve atresia was diagnosed by imaging in all patients except one in whom it could not be distinguished from critical pulmonary stenosis, even with the use of duplex Doppler. Colour Doppler established the diagnosis in this patient by showing no flow across the valve. Colour Doppler showed multiple areas of low velocity flow in the right ventricular myocardium and showed myocardial sinusoids in a patient.

\section{LEFT VENTRICULAR OUTFLOW OBSTRUCTION}

$(\mathbf{n}=15)$

The site of obstruction was identified without the use of colour Doppler in all patients and the maximum gradient was best obtained by nonimaging continuous wave Doppler. The spectral signal of mild aortic regurgitation was noted in five patients (three subaortic stenosis and two aortic stenosis) and colour Doppler identified a very small flow area of trivial aortic regurgitation in four other patients (one subaortic stenosis and three aortic stenosis).

HYPOPLASTIC LEFT HEART SYNDROME $(\mathrm{n}=6)$ Colour Doppler did not influence the detection of mitral atresia $(n=4)$ or aortic atresia $(n=$ 5). In one patient an extremely thin aortic arch was identified only when colour Doppler showed retrograde flow within it. These findings were confirmed at necropsy.

\section{COARCTATION OF THE AORTA $(\mathrm{n}=17)$}

Continuous wave Doppler showed a coarctation type signal from the proximal descending aorta in all patients. The site of narrowing in the descending aorta was identified on imaging in 13 patients. In the other four the images of the descending aorta were inadequate to pinpoint the area of narrowing. Demonstration of proximal acceleration and a narrower area of 
turbulence or variance by colour Doppler identified the site of coarctation in all patients. The three main branches arising from the arch were seen on imaging in every patient and the coarctation was noted to be proximal to the origin of the left subclavian artery in one patient.

TRANSPOSITION OF THE GREAT ARTERIES $(n=11)$ Transposition of the great arteries was diagnosed by imaging in all patients. No patient had a clear defect in the atrial septum but duplex Doppler detected flow through the patent foramen ovale in all patients. Colour Doppler outlined the width of the area of flow through the atrial septum, which gave an indication of mixing between the two circulations.

ARTERIAL DUCTS $(\mathrm{n}=63$ )

A duct was detected without colour Doppler in 62 patients. In the other patient colour Doppler showed a tiny area of continuous flow into the main pulmonary artery and showed a previously undetected duct.

Twenty nine of these 63 patients had a duct dependent pulmonary or systemic circulation; in two of them the duct was not visible on imaging, though ductal flow was detected by spectral Doppler. In these patients colour Doppler outlined the area of flow within the narrow ductal lumen and enabled assessment of the width of the duct.

MITRAL REGURGITATION $(\mathrm{n}=3)$

Colour Doppler was not of additional value in a patient with mild mitral regurgitation associated with mitral valve prolapse and in a patient with severe rheumatic mitral regurgitation and stenosis. In the third patient in whom a ventricular septal defect was suspected on clinical examination, a high velocity systolic spectral signal flowing away from the transducer was misinterpreted as flow through an apical ventricular septal defect. The mitral valve was structurally normal and no ventricular septal defect was seen on imaging. Because colour Doppler showed a small flow jet of mitral regurgitation but no ventricular septal defect we made an unsuspected diagnosis.

\section{OTHER DEFECTS}

The diagnosis of an anomalous left coronary artery was considered in a neonate with poor left ventricular function but both coronary arteries appeared to arise from the aorta and there were no other imaging features associated with the lesion. Colour Doppler was essential for reaching the diagnosis by showing retrograde flow in the left coronary artery and flow into the inferomedial aspect of the main pulmonary artery. Colour Doppler also allowed firm exclusion of this diagnosis in three other infants with dilated cardiomyopathy.

In a patient with a right atrial membrane (cor triatriatum dexter) the large membrane prolapsing into the tricuspid valve orifice was clearly seen on imaging but it was difficult to exclude obstruction with certainty when we used duplex Doppler. Colour Doppler examination in multiple planes confirmed unobstructed flow over the sides of this membrane.

Tricuspid atresia was diagnosed and associated defects assessed in three patients without the use of colour Doppler, which was useful only in outlining the area of flow through an aneurysmal patent foramen ovale in one patient. The other two patients had clear gaps in the atrial septum.

Colour Doppler did not provide any additional information in the assessment of patients with the following defects: common arterial trunk, interrupted aortic arch with a common arterial trunk, anomalous origin of the right pulmonary artery from the aorta, criss-cross arrangement of the atrioventricular valves associated with double outlet right ventricle, cor triatriatum with an obstructed orifice, situs inversus with dextrocardia, unobstructed supracardiac total anomalous pulmonary venous drainage, double inlet left ventricle.

\section{Discussion}

The demonstration of a moving cross sectional image superimposed with real time flow data colour coded for velocity and direction is visually pleasing and enables an easier and faster cardiac ultrasound examination. ${ }^{67}$ This study has not addressed these aspects but has determined the ultrasound information that may be missed if colour Doppler is not used.

In our series of 215 patients colour Doppler was essential for establishing the primary diagnosis in only two patients. The use of colour Doppler $^{8}$ altered the diagnosis from cardiomyopathy to anomalous origin of the left coronary artery and made a very important change in the management of the patient. A patient with minor mitral regurgitation was misdiagnosed as having a probable apical ventricular septal defect on spectral Doppler. (A similar spectral signal of flow away from the transducer was obtained in two other patients in whom colour Doppler confirmed an apical ventricular septal defect.) Review of the echocardiogram suggests that it is likely that the correct diagnosis would have been made if the echocardiographer had not been biased by the provisional diagnosis. Since in practice most echocardiographic examinations are carried out in a goal orientated approach with the clinical diagnosis in mind, colour Doppler may be helpful in highlighting unsuspected defects.

Colour Doppler showed an additional abnormality in several cases in our study. Clinically the most significant of these was the detection of partial anomalous pulmonary venous drainage associated with an atrial septal defect. However, even if this had not been detected and the patient had undergone surgery without cardiac catheterisation the anomalous veins would have been detected and dealt with at operation. As it is often not possible to identify all four pulmonary veins on imaging even in infants with normal pulmonary venous drainage, cross sectional echocardiography cannot be relied upon to exclude partial anomalous pulmonary venous drainage.

Several studies have compared the sensiti- 
vity of colour Doppler with angiography in detecting small or multiple ventricular septal defects. ${ }^{10-12}$ In our study none of the ventricular septal defects visible only with the use of colour Doppler merited further investigations or surgery. Four of these patients underwent surgery or interventional catheterisation for associated lesions (transposition, coarctation, subpulmonary stenosis, pulmonary valve stenosis) without closure of the ventricular septal defect.

Colour Doppler can be useful in the assessment of atrial septal defects in adults ${ }^{13}$ but in children the defect is usually clearly seen on imaging. However, in the absence of a clear atrial septal defect, colour Doppler is useful in showing the width of the flow area through the septum in patients with cyanotic heart defects who are dependent on atrial shunting.

The assessment of severity of valve regurgitation by colour Doppler is a contentious issue because the extent of the colour jet is much more dependent on the driving pressure of regurgitation than on its volume. ${ }^{14}$ The simultaneous display of the spatial orientation of different jets is nevertheless useful in detecting the presence of left ventricle to right atrium regurgitation in atrioventricular septal defects. Colour Doppler is extremely sensitive in detecting valve regurgitation ${ }^{15}$ and the detection of trivial aortic regurgitation in four additional patients with aortic or subaortic stenosis was of no clinical importance. Localisation of the jet of tricuspid regurgitation by colour Doppler, however, can sometimes improve the spectral Doppler waveform enabling right ventricular pressure estimation.

Although the proximal parts of the right and left pulmonary arteries are seen on imaging, the distal parts are more difficult to visualise because they are often obscured by lungs. In our series colour Doppler enabled visualisation of normal sized distal right and left pulmonary arteries $(\mathrm{n}=4)$, a stenotic distal pulmonary artery $(n=1)$, a hypoplastic pulmonary artery $(\mathbf{n}=1)$, and bilateral peripheral pulmonary stenosis $(n=1)$. The impact of improved definition with colour Doppler echocardiographic examination of distal pulmonary artery branch anatomy in tetralogy of Fallot may be less important in clinical practice because in most centres all patients with tetralogy of Fallot undergo cardiac catheterisation before total repair.

In a recent comparative study of colour Doppler and angiography in the assessment of pulmonary atresia with ventricular septal defect Smyllie et al concluded that colour Doppler could be used reliably to assess patients with confluent pulmonary arteries and a unifocal blood supply. ${ }^{16}$ In our series colour Doppler was useful in distinguishing pulmonary valve atresia from critical pulmonary stenosis in two infants. In critical pulmonary stenosis the tiny orifice in the dysplastic pulmonary valve may not be clearly visible on imaging $^{17}$ and with spectral Doppler flow through it may be difficult to distinguish from ductal flow. This differentiation could be useful because pulmonary valvotomy may be contemplated in addition to a shunt procedure.
The presence of a coarctation can be readily detected by continuous wave Doppler but it is not always possible to image the site of narrowing because the descending aorta may be obscured by lungs. Colour Doppler can show the precise site of narrowing in these patients, which may not be relevant to the method of surgical repair but nevertheless may reduce the need for cardiac catheterisation in cases of doubt.

Although it has been reported that colour Doppler facilitates maximum velocity measurement in valve stenosis by enabling optimum alignment of the Doppler beam, ${ }^{18}$ in our series colour Doppler did not show a higher gradient in any patient with left or right ventricular outflow obstruction or coarctation. Colour Doppler merely shows the component of a jet in a two dimensional plane, and because it is not possible to predict the true direction of the jet the maximum velocity is best obtained by Doppler examination from several sites at varying angles.

It is difficult to be totally objective in the analysis of a comparative study of ultrasound methods because echocardiography is largely an interpretative process involving operator interaction. Hence the apparent deficiency of a technique to elucidate a diagnosis may lie in the operator's inability to assimilate and interpret the available information. This was apparent in the case of mild mitral regurgitation which was misdiagnosed as apical ventricular septal defect and also in a case of left heart hypoplasia in which a very thin aorta was identified only by using colour Doppler during the study but was clearly visible on imaging to another operator at a subsequent examination.

\section{CLINICAL IMPLICATIONS}

Our study shows that most patients with congenital heart disease can be diagnosed by imaging and duplex Doppler echocardiography with colour Doppler providing little additional information. The use of colour Doppler ensured appropriate surgical treatment for a patient with an anomalous coronary artery and led to preoperative cardiac catheterisation in a patient with anomalous pulmonary venous drainage. Although colour Doppler influenced the final non-invasive diagnosis in several other patients by providing further information on the diagnosis or by showing an additional defect it did not alter the course of conservative or surgical management of these patients. Such information is nevertheless important because it increases confidence in referring patients for surgery without invasive investigations. Ideally all patients should undergo a colour Doppler study before cardiac surgery because there is a very small but unavoidable risk of missing an important defect. Colour Doppler studies must also be performed on all patients with dilated cardiomyopathy of uncertain origin including those diagnosed in the past before colour Doppler became available.

We recommend that all centres undertaking major paediatic cardiac surgery should have the facility for colour Doppler studies. In 
paediatric cardiology centres in countries with limited financial resources, however, machines without colour Doppler can provide an adequate service because colour Doppler does not alter the management of most patients with congenital cardiac defects. Machines without colour Doppler are also quite adequate for the assessment of congenital heart defects in district hospitals with regular paediatric cardiology clinics and in neonatal units.

JPG is in receipt of a grant from the Equipment Evaluation Committee of the Scottish Home and Health Department.

1 Gutgesell HP, Huhta JC, Latson LA, Huffines D, McNamara D. Accuracy of two-dimensional echocardiography in the diagnosis of congenital heart disease. $\mathrm{Am} \mathrm{J}$ Cardiol 1985;55:514-8.

2 Leung MP, Mok CK, Lau KC, Lo R, Yeung CY. The role of cross sectional echocardiography and pulsed Doppler ultrasound in the management of neonates in whom congenital heart disease is suspected: a prospective study. Br Heart J 1986;56:73-82.

3 Miyatake $\mathrm{K}$, Okamoto $\mathbf{M}$, Kinoshita $\mathrm{N}$, et al. Clinical applications of a new type of real-time two-dimensiona Doppler flow imaging system. Am J Cardiol 1984;54: $857-68$.

4 Huhta JC, Glasow P, Murphy DJ, et al. Surgery without catheterization for congenital heart defects: management of 100 patients. J Am Coll Cardiol 1987;9:823-9.

5 Leung MP, Cheung DLC, Lo RNS, Mok CK, Lee J, Yeung $\mathrm{CY}$. The management of symptomatic neonates with CY. The management of symptomatic neonates with
suspected congenital heart disease using combined crosssuspected congenital heart disease using combined cross-
sectional echocardiography and pulsed Doppler flow study sectional echocardiography and pulsed Doppler flow study
as the definitive investigations. Int J Cardiol 1989;24:41-6.

6 Goldberg HJ. A perspective on color-coded Doppler echocardiography: utility or just another pretty picture? J Am

7 Monaghan MJ, Mills P. Doppler colour flow mapping: technology in search of an application? Br Heart $J 1989$; 61:133-8.

8 Houston AB, Pollock JCS, Doig WB, et al. Anomalous origin of the left coronary artery from the pulmonary trunk: elucidation with colour Doppler flow mapping. Br Heart J 1990;63:50-4.

9 Sahn DJ, Allen HD, Lange LW, Goldberg SJ. Crosssectional echocardiographic diagnosis of the sites of total anomalous pulmonary venous drainage. Circulation 1979; 60:1317-25.

10 Sutherland GR, Smyllie JH, Ogilvie BC, Keeton BR. Colour flow imaging in the diagnosis of multiple ventricular septal defects. Br Heart J 1989;62:43-9.

11 Chin AJ, Alboliras ET, Barber G, et al. Prospective detection by Doppler color flow imaging of additional defects in infants with a large ventricular septal defect. J Am Coll Cardiol 1990;15:1637-42.

12 Ortiz E, Robinson PJ, Deanfield JE, Franklin R, Macartney FJ, Wyse RKH. Localisation of ventricular septal defects by simultaneous display of superimposed colour Doppler
and cross sectional echocardiographic images. Br Heart J and cross section

13 Suzuki Y, Kambara H, Kadota K, et al. Detection of intracardiac shunt flow in atrial septal defect using a realtime two-dimensional color-coded Doppler flow imaging system and comparison with contrast two-dimensional echocardiography. Am J Cardiol 1985;56:347-50.

14 Simpson IA, Valdes-Cruz LM, Sahn DJ, Murillo A, Tamura T, Chung KJ. Doppler color flow mapping of simulated in vitro jets: evaluation of the effects of orifice size and haemodynamic variables. J Am Coll Cardiol 1989;13: 1195-207.

15 Yoshida K, Yoshikawa J, Shakudo M, et al. Colour Doppler evaluation of valvular regurgitation in normal subjects. Circulation 1988;78:840-7.

16 Smyllie JH, Sutherland GR, Keeton BR. The value of Doppler color flow mapping in determining pulmonary blood supply in infants with pulmonary atresia with ventricular septal defect. $J$ Am Coll Cardiol 1989;14: ventricular

17 Silove ED, de Giovanni JV, Shiu MF, Yi MM. Diagnosis of right ventricular outflow obstruction in infants by cross right ventricular outflow obstruction in infants by cross
sectional echocardiography. Br Heart $J$ 1983;50:416-20. 18 Pandian NG, Thanikachalam S, Elangovan D, Caldeira ME,
Salem DN. Colour Doppler flow imaging in valvular stenosis. Echocardiography 1987;4:515-26. 